brido Filtek Z100. Os dentes foram seccionados nas direções X e Y de forma a obter palitos com secção transversal de $1 \pm 0,2$ $\mathrm{mm} 2$. Os palitos obtidos de cada dente foram aleatoriamente divididos em 2 subgrupos e armazenados em água destilada durante 24 horas ou 6 meses. Após o período de envelhecimento, forma realizados os testes de resistência adesiva a tensões de tração numa máquina de testes universal (Shimadzu; 1 $\mathrm{mm} / \mathrm{min} ; 1 \mathrm{KN}$ ) até ocorrer fratura. O valor médio da resistência adesiva obtida nos diversos palitos de cada dente foi utilizado como valor desse dente, considerado como unidade experimental. A superfície de fratura foi observada em estereomicroscópio e o tipo de falha de união foi classificado em adesivo, coesivo ou misto. Os dados de microtração foram analisados estatisticamente com ANOVA de medidas repetidas seguido de testes post-hoc segundo Dunnett $(\alpha=0,05)$. Resultados: Os valores médios de resistência adesiva variaram entre 12,9 MPa para o grupo IE1 (24 h) e 24,0 MPa para o grupo CHX 0,2 (6 m). O tempo de envelhecimento não influenciou de forma estatisticamente significativa $(p=0,361)$ a resistência adesiva, nem se observou interação significativa $(p=0,230)$ entre inibidores e envelhecimento. Apesar de se terem observado diferenças estatisticamente significativas $(p=0,029)$ entre os inibidores, não foram identificadas diferenças estatisticamente significativas $(p>0,05)$ entre os diversos inibidores e o Optibond FL sem inibidores. A falha foi predominantemente do tipo adesivo (91,8\%). Conclusões: A aplicação prévia de inibidores de MMPs não influenciou a resistência adesiva ao longo de 6 meses.

http://doi.org/10.24873/j.rpemd.2020.12.804

\section{\#082 A Prevalência de Cárie Dentária e o Risco Erosivo em Atletas - Revisão Sistemática}

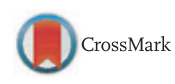

Daniela Jácome*, Cecília Rozan, Filipa Vicente, Carlos Família, André Júdice, Catarina Godinho

Instituto Universitário Egas Moniz; Grupo de Patologia Médica Nutrição e Exercício Clínico do CiiEM; Laboratório de Patologia Molecular do Centro de Investigação Interdisciplinar Egas Moniz

Objetivos: Avaliar e analisar o conhecimento atual sobre a prevalência da cárie dentária e risco erosivo em atletas e determinar o papel da alimentação e prática desportiva nestes processos patológicos, para esta população. Materiais e métodos: Foram utilizadas as bases de dados Pubmed, Cochrane Library e B-on para realizar uma pesquisa bibliográfica com base nas palavras chave 'Dental Caries', "Tooth Erosion”, "Sports Medicine" "Diet". Os artigos resultantes desta pesquisa foram selecionados com base em critérios de inclusão e exclusão bem definidos, entre os quais a utilização do índice de dentes cariados perdidos e obturados por dente/superfície (CPOD/CPOS) e/ou o índice Internacional Caries Detection and Assessment (ICDAS) para de determinação da prevalência de cárie, e a utilização do índice Basic Erosive Wear Examination (BEWE) para a determinação do risco erosivo. Resultados: De um total de 188 artigos resultantes da pesquisa bibliográfica, apenas 6 reuniram todos os requisitos para inclusão neste estudo. Estes mostram que os valores de prevalência de cárie dentária se encontram entre os $36,9 \%$ e os $92,5 \%$ e os valores de erosão dentária entre os $40 \%$ e os $53,1 \%$ o que está associado a um risco de erosão dentária moderado. No entanto, nenhum dos estudos analisado mostrou haver uma relação significativa entre a prevalência de cárie e risco erosivo com a alimentação dos atletas. Conclusões: A prática desportiva apresenta um impacto significativo na saúde oral dos atletas, o que por sua vez influencia a sua performance desportiva. Muitas vezes este impacto ocorre ao nível da prevalência de cárie dentária e risco erosivo, que fora do contexto desportivo, se sabe serem influenciados de forma significativa pelo comportamento alimentar de cada indivíduo. No entanto a informação existente relativa a atletas é escassa e estes parâmetros são determinados de forma não estandardizada. Isto impede-nos de perceber qual a real influencia das dietas características desta população ao nível da saúde oral dos atletas. Desta forma é essencial a padronização dos métodos de avaliação da prevalência de cárie dentária, risco erosivo e do comportamento alimentar em atletas.

http://doi.org/10.24873/j.rpemd.2020.12.805

\#083 Caracterização dos determinantes que levam á procura de cuidados de saúde na gravidez

Marta Fernandes Ramos*, Maria de Lurdes Lobo Pereira

Faculdade de Medicina Dentária da Universidade do Porto (FMDUP)

Objetivos: O objetivo deste estudo é caracterizar os fatores sociodemográficos que podem influenciar a presença de problemas orais na gravidez bem como na procura de cuidados de saúde oral durante este período. Materiais e métodos: Avaliaram-se os dados sociodemográficos e comportamentos maternos relacionados com a saúde oral durante a gravidez. Estes dados foram extraídos do estudo de coorte pertencente ao Projeto Geração XXI, Nascer e Crescer no Milénio, realizado em 2005-2006. Utilizou-se regressão logística para avaliar quais os fatores sociodemográficos que implicavam na visita ao médico dentista e uma outra para avaliar quais os fatores sociodemográficos que implicavam na prevalência de problemas orais durante a gravidez. Resultados: A maioria das mulheres, $85,6 \%$, não consultou o médico dentista durante a gravidez e $28,8 \%$ reportou dor dentária ou dor ao mastigar. Dessas apenas $26,4 \%$ visitaram o médico dentista nesse período. Relativamente à presença de problemas orais durante a gravidez verificou-se que grávidas não ativas profissionalmente apresentam uma maior probabilidade de terem problemas orais $(\mathrm{OR}=1,38$; $\mathrm{IC}=1,186$ - 1,593). Relativamente à idade e escolaridade, verificou-se que à medida que o nível destas variáveis aumenta menor é a probabilidade das grávidas terem problemas orais neste período. A escolaridade elevada $(\mathrm{OR}=1,59 ; \mathrm{IC}=1,287-1,969)$ e rendimento mensal elevado $(\mathrm{OR}=1,49 ; \mathrm{IC}=1,211-1,832)$ da gestante afetam positivamente a visita ao médico dentista durante a gravidez. Quanto mais baixa é a posição socioeconómica da mãe aos 12 anos menor a probabilidade de visitar o médico dentista durante a gravidez. Conclusões: É fundamental incutir precocemente a importância dos cui- 
dados de saúde oral no decorrer do trajeto de vida, contudo na gravidez este aspeto reveste-se de particular importância. Esta fase é um período sensível no qual existem várias alterações, nomeadamente na cavidade oral, e como tal deve ser englobada na saúde materna geral, uma vez que das alterações na saúde oral da grávida podem advir consequências para o feto. É importante criar condições para reduzir as desigualdades na procura de cuidados de saúde oral durante a gravidez e a criação de equipas multidisciplinares que incluam os profissionais de saúde oral pode contribuir para esta redução.

http://doi.org/10.24873/j.rpemd.2020.12.806

\section{\#084 Caraterização da Qualidade de Vida} Relacionada com a Saúde Oral em Adolescentes de 18 Anos

Mariana Azevedo Melo*, Mariana Rodrigues Viana da Silva Pereira, Maria Cristina dos Santos de Sousa Ferreira, Maria de Lurdes Ferreira Lobo Pereira

Direção Geral da Saúde; Faculdade de Medicina Dentária da Universidade do Porto

Objetivos: Estudar o impacto dos fatores sociodemográficos e das condições de saúde oral, nomeadamente as necessidades de tratamento não satisfeitas no que concerne à cárie dentária, os dentes perdidos e a presença de traumatismos dentários na Qualidade de Vida Relacionada com a Saúde Oral de uma população portuguesa de adolescentes de 18 anos. Materiais e métodos: Foram utilizados dados secundários do terceiro Estudo Nacional de Prevalência de Doenças Orais, referentes a adolescentes portugueses de 18 anos. Os dados foram recolhidos a partir de um questionário relativo aos fatores sociodemográficos e aos impactos da saúde oral na qualidade de vida, para além de um exame clínico à cavidade oral dos adolescentes. A análise estatística foi realizada através do programa SPSS, versão $25\left(\right.$ IBM $\left.^{\circledR}\right)$. Resultados: Verificou-se que $32,7 \%$ dos adolescentes reportaram pelo menos um impacto da saúde oral na qualidade de vida nos últimos 12 meses. Constatou-se que o sexo e a atividade laboral dos adolescentes influenciam significativamente os impactos na qualidade de vida relacionada com a saúde oral. Demonstrou-se, ainda, que a existência de necessidades de tratamento não satisfeitas quanto à cárie dentária, de dentes perdidos e de traumatismos dentários também tem uma influência significativa na qualidade de vida, originando mais impactos negativos nesta. Conclusões: A existência de impactos negativos na Qualidade de Vida Relacionada com a Saúde Oral varia com o estado da saúde oral e os fatores sociodemográficos dos adolescentes. Nas consultas de Medicina Dentária devem ser tomadas em consideração as preocupações que os adolescentes demonstram com a sua saúde oral, bem como os problemas orais que originam impactos físicos, sociais e psicológicos na sua vida diária, de modo a proceder à sua identificação e resolução, contribuindo consequentemente para uma melhoria da sua Qualidade de Vida.

http://doi.org/10.24873/j.rpemd.2020.12.807
\#085 Atitudes, comportamentos e estado de saúde oral de alunos do $5 .^{\circ}$ ano de Medicina Dentária

Sandrine Almeida*, Teresa Albuquerque, Sónia Mendes

Faculdade de Medicina Dentária da Universidade de Lisboa

Objetivos: Este estudo pretendeu: 1) Conhecer as atitudes e comportamentos de saúde oral dos estudantes do último ano do curso de Medicina Dentária; 2) Estudar o estado de saúde oral e sua autoperceção; 3) Relacionar as atitudes e comportamentos com o estado de saúde oral e 4) Conhecer as crenças sobre as atitudes e comportamentos. Materiais e métodos: A população-alvo foi constituída pelos estudantes do $5 .^{\circ}$ ano do curso de Mestrado Integrado de Medicina Dentária da Faculdade de Medicina Dentária da Universidade de Lisboa. Os dados foram recolhidos por um questionário sobre as atitudes e comportamentos de saúde oral, incluindo o Hiroshima University Dental Behavioural Inventory (HUDBI) e por um exame intraoral para o estudo de cárie (ICCMS), do nível de higiene oral (ID-S) e da inflamação gengival (IPC modificado). Foi realizada a estatística descritiva e utilizados os testes, Mann-Whitney, Kruskal-Wallis e correlação de Spearman $(\alpha=0,05)$. Resultados: O valor médio de HUDBI foi 9,20 ( $d p=1,34)$. Quase todos os estudantes $(97,8 \%)$ escovavam os dentes bidiariamente, mas apenas $23,9 \%$ utilizavam o fio dentário diariamente. A prevalência de cárie foi 100\%, sendo o CA-6POD médio 10,4 ( $\mathrm{dp}=4,2)$. O nível de higiene oral foi excelente em $43,5 \%$ dos estudantes. A frequência de inflamação gengival foi de $100 \%$, sendo o IPC modificado médio $0,37(\mathrm{dp}=0,2)$. Apenas se verificou uma associação positiva entre o consumo de alimentos açucaradas e o C3-6POD $(p=0,03)$. Os estudantes referiram que a importância de comportamento saudáveis foi influenciada pelo conhecimento das doenças orais adquirido no curso e que a dificuldade do uso diário do fio dentário é explicada devido à técnica difícil, ao tempo exigido, cansaço e preguiça. Conclusões: Os estudantes apresentaram atitudes e comportamentos positivos e bons indicadores de higiene oral e inflamação gengival, contudo, a prevalência de cárie foi elevada.

http://doi.org/10.24873/j.rpemd.2020.12.808

\#086 Qualidade de vida relacionada com a saúde oral dos estudantes da Universidade de Lisboa

Mónica Chantre*, Mário Bernardo, Sónia Mendes

Faculdade de Medicina Dentária da Universidade de Lisboa

Objetivos: A qualidade de vida relacionada com a Saúde Oral (QdVRSO) é uma parte integrante da saúde e bem-estar que visa avaliar o impacto da saúde oral em aspetos da vida pessoal e social. O estudo teve como objetivos estudar a QdVRSO e relacioná-la com os comportamentos sociodemográficos e académicos, os comportamentos de saúde oral, a presença de problemas orais e a autopercepção de saúde oral. Materiais e métodos: A recolha de dados foi realizada através da distribuição de um questionário online composto por 30 perguntas, que incluiu características so- 